\title{
Performance of randomized Kelvin cell structures as catalytic substrates: Mass-transfer based analysis
}

\author{
Francesco Lucci $^{\mathrm{a}, *}$, Augusto Della Torre ${ }^{\mathrm{b}}$, Jan von Rickenbach ${ }^{\mathrm{a}, \mathrm{c}}$, Gianluca Montenegro ${ }^{\mathrm{b}}$, Dimos \\ Poulikakos $^{c}$, Panayotis Dimopoulos Eggenschwiler ${ }^{\mathrm{a}}$ \\ a Laboratory for I.C. Engines, Empa, Swiss Federal Laboratories for Materials Testing and Research, Dübendorf, Switzerland \\ ${ }^{\mathrm{b}}$ Dipartimento di Energia, Politecnico di Milano, Milano, Italy \\ c Laboratory of Thermodynamics in Emerging Technologies, Swiss Federal Institute of Technology, ETH, Zürich, Switzerland
}

- We perform a numerical study of the mass transfer in Kelvin cell structures.

- We reach qualitative agreement with experimental works on foams.

- New correlations are presented.

- The Lévêque analogy is verified.

A R T I C L E I N F O

Article history:

Received 23 December 2013

Received in revised form 12 March 2014

Accepted 24 March 2014 Available online 29 March 2014

\section{A B S T R A C T}

Open cell foams are attractive materials for various industrial applications, but building accurate universal correlations is challenging due to their great geometrical complexity. Momentum and mass transfer of a randomly packed Kelvin cell structures are numerically investigated. Porosity, $\varepsilon$, Kelvin cell size, PPI, and inflow velocity, $u$, are systematically varied for a total of 120 simulations. Correlations for geometrical and transfer properties are discussed. The analogy based on the generalized Lévêque equation (Martin, 2002) between mass and momentum transfer is evaluated and it is qualitatively in agreement with the results of Incera Garrido et al. (2008) on ceramic foams.

Keywords:

Kelvin cell

Foam

Sherwood number

Mass transfer

Catalyst

\section{Introduction}

Open cell foams are attractive materials characterized by high porosity, low density, high mechanical strength and large surface area that in the recent years have been investigated for various industrial applications like filters, heat exchangers and catalytic reactors. As a catalyst support they present several advantage over monoliths and packed beds. The open cell structure allows higher flow uniformity which is a critical factor for the pollutant conversion efficiency and for the catalyst durability (Gaiser et al., 2003; Martin et al., 2008; Zygourakis, 1989). The tortuous flow path is expected to enhance the mixing and the heat/mass transfer. The high specific surface will yield more compact catalysts (Giani et al., 2005a).

In the field of automotive catalysts a critical parameter is the pressure drop, which affects engine efficiency. Foams have higher pressure drop then a monolith with the same dimensions (Twigg and Richardson, 2007). This can be compensated by an increased mass transfer that allows us to downsize the catalyst (Dimopoulos Eggenschwiler et al., 2009) or by different geometrical reactor configurations (Koltsakis et al., 2008).

Till now, numerous works have investigated foam performances but due to their great variability in geometrical and chemical properties the results of the investigations still do not converge among different authors. The absence of accepted correlations, on one side, makes it difficult for engineers to adopt such an innovative catalyst support and, on the other, proves the need for further investigations.

In a recent literature review Edouard et al. (2008) concluded that no pressure drop correlation produced consistently good results and that standard deviation with experimental values can be as high as 100\%. Between the analyzed correlations they 
reported that the most promising are the ones proposed by Du Plessis et al. (1994) and Lacroix et al. (2007). A similar uncertainty can be made about studies on heat and mass transfer (Richardson et al., 2003; Giani et al., 2005b; Incera Garrido et al., 2008; Huu et al., 2009).

In order to simplify the analysis, foams have been modelled as regular structures. The most used geometric cells are a cubic cell (Du Plessis et al., 1994; Evans et al., 1998; Giani et al., 2005b) or a tetrakaidecahedron, also called as a Kelvin cell (Richardson et al., 2000; Inayat et al., 2011a; Bai and Chung, 2011). Other studies tried to improve the model with similar structures (Boomsma et al., 2003; Huu et al., 2009). Inayat et al. (2011b) presented also some experimental analysis of a self-manufactured Kelvin cell structure. These regular geometries are used to derive expression for foam properties, like the specific surface area (SSA), which are difficult to derive experimentally (Richardson et al., 2000; Giani et al., 2005a; Inayat et al., 2011a); or as a model for analytical analysis (Du Plessis et al., 1994; Fourie and Du Plessis, 2002; Ahmed et al., 2011); or as a geometry for computational analysis (Boomsma et al., 2003; Krishnan et al., 2006; Habisreuther et al., 2009; Bai and Chung, 2011). Numerical simulations up to now have mainly used to study the foam pressure drop or the heat transfer properties. Little work has been done for the simulation of the mass transfer.

The present work is a parametric CFD study of Kelvin cell structures. Its purpose is to study foams in a controlled environment to help understand which are the most important parameters affecting their performances.

\section{Method}

The study is performed over a structure of $4 \times 2 \times 2$ randomized Kelvin cells (KC). Porosity $(\varepsilon=0.78,0.82,0.86,0.89)$, Kelvin cell size (PPI $=11,14,22,28,44)$ and inflow velocity $(u=1.0,2.5$, $5.0,7.5,10,15 \mathrm{~m} / \mathrm{s}$ ) are systematically varied for a total of 120 simulations. The resulting Reynolds number based on the external pore diameter $D_{p}$ is included in the range [7:470]. In Fig. 1 a Kelvin cell is shown with its characteristic dimensions and in Table 1 we report a summary of the geometrical properties of the Kelvin cell structures used.

\subsection{Domain}

The computational domain is built starting from a 3D CAD model generated using the open source software Salome. The model consists of a regularly stacked structure of Kelvin cells: 4 cells in the flow direction and 2 cells in the direction perpendicular to the flow. Struts are assumed to be cylindrical. A randomization is performed by randomly moving each node of the

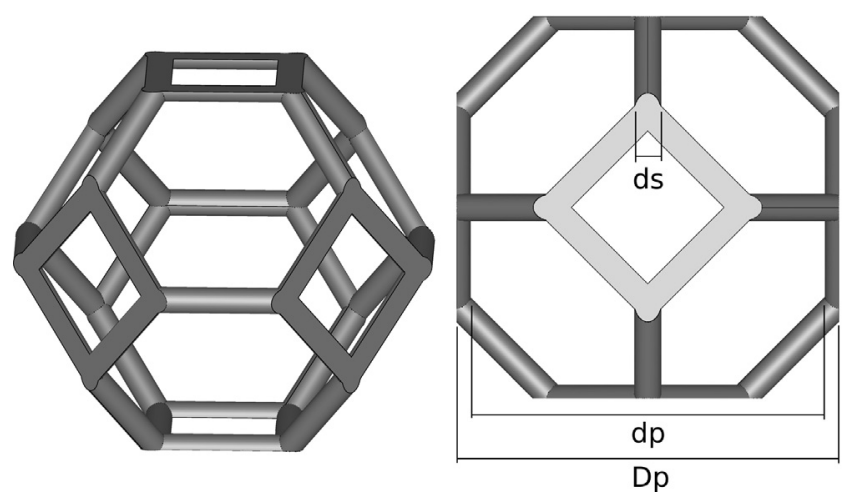

Fig. 1. Kelvin cell with characteristic dimensions.
Table 1

Geometric properties of KC structures simulated.

\begin{tabular}{lllll}
\hline$\varepsilon$ & PPI & SSA $\left(\mathrm{m}^{2} / \mathrm{m}^{3}\right)$ & $d_{p}(\mathrm{~mm})$ & $d_{s}(\mathrm{~mm})$ \\
\hline 0.80 & 11 & 1500 & 1.85 & 0.45 \\
0.80 & 14 & 1876 & 1.48 & 0.36 \\
0.80 & 22 & 3001 & 0.925 & 0.225 \\
0.80 & 28 & 3751 & 0.74 & 0.18 \\
0.80 & 44 & 6002 & 0.4625 & 0.1125 \\
0.84 & 11 & 1399 & 1.9 & 0.4 \\
0.84 & 14 & 1748 & 1.52 & 0.32 \\
0.84 & 22 & 2797 & 0.95 & 0.2 \\
0.84 & 28 & 3496 & 0.76 & 0.16 \\
0.84 & 44 & 5594 & 0.475 & 0.1 \\
0.87 & 11 & 1283 & 1.95 & 0.35 \\
0.87 & 14 & 1603 & 1.56 & 0.28 \\
0.87 & 22 & 2566 & 0.975 & 0.175 \\
0.87 & 28 & 3207 & 0.78 & 0.14 \\
0.87 & 44 & 5131 & 0.4875 & 0.0875 \\
0.90 & 11 & 1152 & 2.0 & 0.3 \\
0.90 & 14 & 1440 & 1.6 & 0.24 \\
0.90 & 22 & 2304 & 1.0 & 0.15 \\
0.90 & 28 & 2881 & 0.8 & 0.12 \\
0.90 & 44 & 4609 & 0.5 & 0.075 \\
\hline
\end{tabular}

regular structure in a procedure similar to Habisreuther et al. (2009). The vector $\boldsymbol{x}_{i}$ of the position of the node $i$ in the regular structure can be expressed as

$\boldsymbol{x}_{i}=\boldsymbol{x}_{i}^{\complement}+\boldsymbol{x}_{i}^{f}+\boldsymbol{x}_{i}^{n}$

where $\boldsymbol{x}_{i}^{C}$ is the position of the center of the cell, $\boldsymbol{x}_{i}^{f}$ is the relative position of the KC quadrilateral face relative to the KC center and $\boldsymbol{x}_{i}^{f}$ is the relative position of node from the center of the face. The position of the randomized node will be

$\boldsymbol{x}_{i}=\boldsymbol{x}_{i}^{C}+\left(\boldsymbol{x}_{i}^{f}+\alpha \boldsymbol{r}_{i}^{f}\right)+\left(\boldsymbol{x}_{i}^{n}+\alpha \boldsymbol{r}_{i}^{n}\right)$

where $\alpha$ is a fixed scalar equal to $40 \%$ of the strut length $\alpha=0.4 L_{s}$ and $\boldsymbol{r}_{i}^{f}, \boldsymbol{r}_{i}^{n}$ are random vectors of intensity less than 1 . Note that $\boldsymbol{r}_{i}^{f}$ is kept constant for all nodes on the same quadrilateral face. "Gost" struts are created outside the domain in order to maintain the periodicity of the structure. A sample of the structure can be seen in Fig. 2. The porosity of the structure is controlled by the ratio of diameter to length of the strut.

Once the 3D model is created, a grid mesh is generated, using the blockMesh and snappyHexMesh tools. A first hexahedral mesh was generated by blockMesh. The inflow plane is placed upstream of the KC structure at a distance of about $1 / 2$ the average cell length. The outflow plane was placed approximately a full cell length downstream the $\mathrm{KC}$ structure in order to minimize the effect of the strut wakes on the outflow conditions. Using snappyHexMesh the grid is refined at the fluid-solid boundary with two castellated levels. Finally cell vertexes close to the boundary are snapped to the boundary. In the process some polyhedral cells are created. The final computational grid is made of approximately $4.5 \times 10^{5}$ cells, of which $0.9 \times 10^{5}$ are not hexahedrons. The average size of the cells close to the fluid-solid interface is approximately $1 / 10$ of the strut diameter. A sample of the final grid can be seen in Fig. 3, another view of the surface refinement can be seen in Fig. 2.

\subsection{Numerical modelling and assumptions}

No transient conditions are considered and the catalyst is assumed to have reached a steady state. The SIMPLE algorithm is implemented in a modified version of reactingFOAM, a standard OpenFOAM solver. 

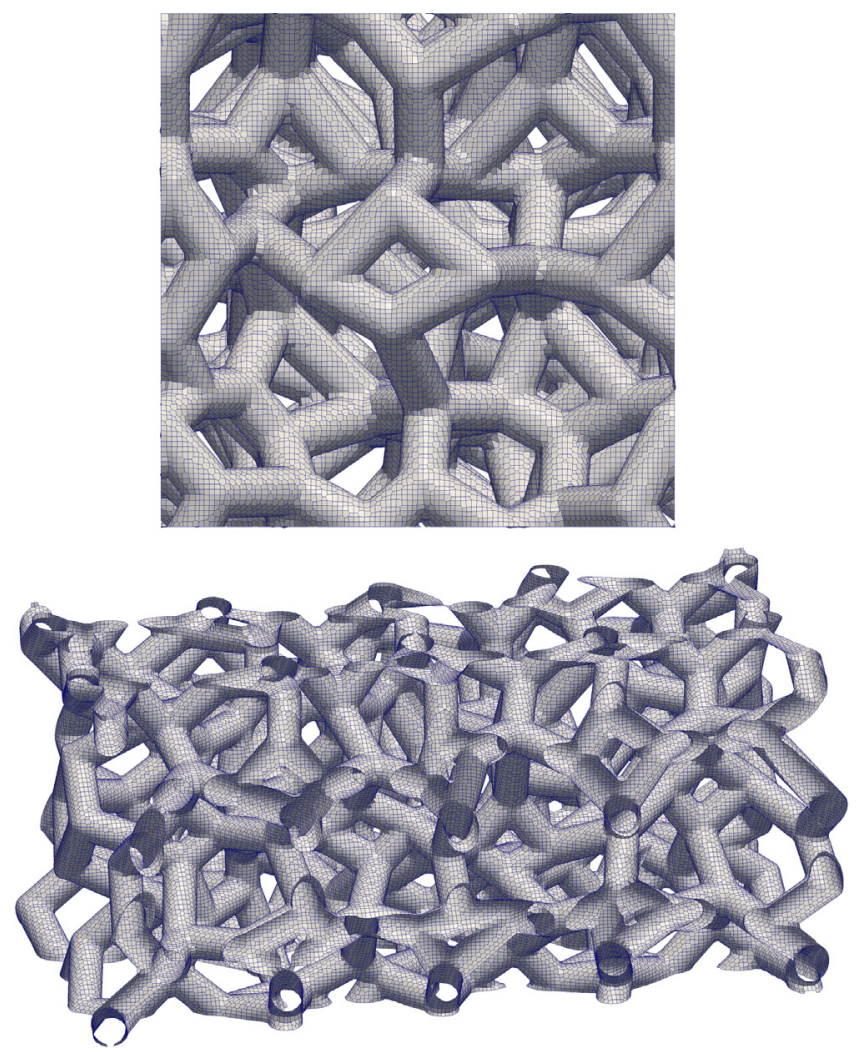

Fig. 2. Sample view of randomized Kelvin cell structure. Grid resolution is plotted on the foam surface.

We simulate the transport of methane $\mathrm{CH}_{4}$ in air. A Sutherland model is applied for the transport air properties and the thermal properties are extracted from Janaf tables. The methane inflow mass concentration is $X_{\mathrm{CH} 4}=0.001$ and is assumed to have Smidth number equal to 1 .

The catalyst operates in a transport limited regime so the temperature at the inflow is kept constant at $700 \mathrm{~K}$. No conjugate heat transfer between the solid and the fluid is solved but a constant temperature of $750 \mathrm{~K}$ is imposed at the solid-fluid interface. The assumption was verified by tests with conjugate heat transfer and a multi-region solver, which showed a difference between the maximum and the minimum temperature in the solid matrix of less than $3^{\circ}$.

Gas phase reactions are neglected. Infinite fast heterogeneous reactions are modelled at the solid-fluid interface as boundary condition imposing

$X_{\mathrm{CH} 4}=0$

and

$\frac{\partial X_{i}}{\partial \mathbf{n}}=\alpha_{i} \frac{M_{i}}{M_{C H 4}} \frac{\partial X_{C H 4}}{\partial \mathbf{n}}$

where $\alpha_{i}$ is the stoichiometric coefficient of species $i$.

At the $x^{-}$boundary are applied fixedvalue inflow conditions, at the $x^{+}$boundary inletoutlet conditions and cyclic conditions are applied in the $y$ and $z$ directions.

\section{Results and discussion}

\subsection{KC structures versus open cell foams}

In this section we compare our results with correlations derived by groups working with catalytically reacting foams and

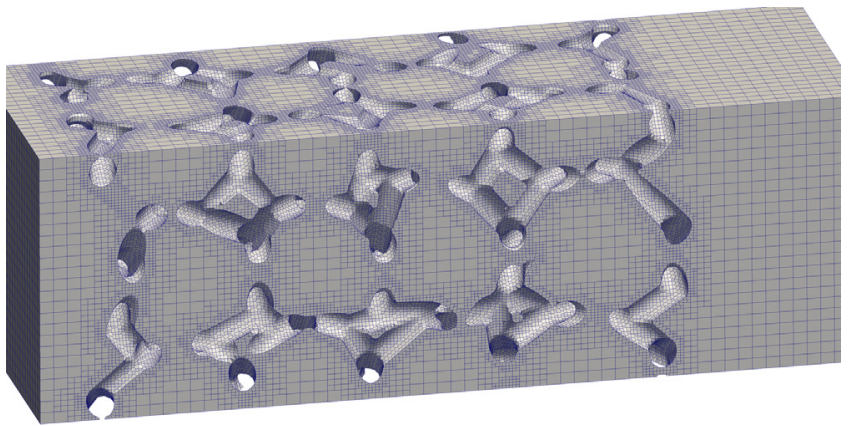

Fig. 3. Sample of the final computational grid.

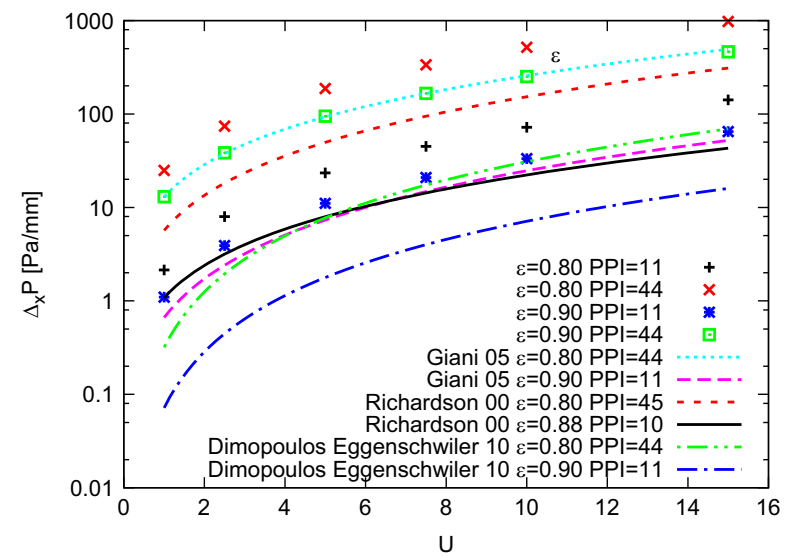

Fig. 4. Pressure drop per unit length in $\mathrm{Pa} / \mathrm{mm}$ versus inflow velocity for selected cases. Lines: literature correlations; Symbols: present work.

that are most used in the literature. Note that each author uses a different characteristic length scale and there is no agreement on the definition of pore diameter. Each correlation plotted in the present work is computed using the characteristic length of each respective author. Then it is rescaled to be consistent with the characteristic length of the external KC diameter $D_{p}$.

In Fig. 4 the pressure drop is plotted over the inflow velocity. Our results for $\varepsilon=0.78$ and 0.89 and $P P I=11$ and 44 (symbols) are compared with correlations from Richardson et al. (2000), Giani et al. (2005a) and Dimopoulos Eggenschwiler et al. (2009). Our KC simulations predict an higher pressure drop than all the correlations, but they follow their trend. The correlation from Giani at al. is the one that better follows our results for both extremes of the data set (I.E. $[\varepsilon, P P I] \sim[0.78,11]$ and $[0.89,44])$.

The predicted mass transfer by our KC simulation is higher that the one published in the literature for real foams. In Fig. 5 the Sherwood number for all sets of data is compared with correlations from Richardson et al. (2000), Giani et al. (2005a) and Incera Garrido et al. (2008). For Reynolds numbers lower than 100 the slope of our results in a log-log plot is consistent with Groppi et al. (2007) and with Incera Garrido et al. (2008), while at higher Reynolds number a better agreement is reached with Richardson. Overall the best fit is obtained by the correlation of Groppi et al. (2007), which underestimates our results by less than $20 \%$.

One of the purposes of this work is to identify critical parameters and characteristics of open cell structures to help design sound and reliable physical models for real foams. Thus it is useful to understand the reasons for the differences between $\mathrm{KC}$ structures and real foams.

As mentioned in Section 1, the pressure drop and mass transfer correlations published in the literature present a great variability. Predictions can differ even one order of magnitude among 


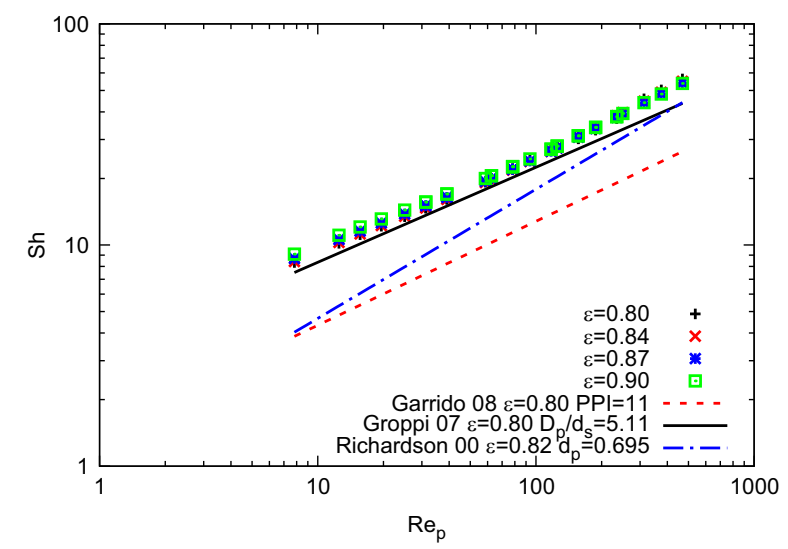

Fig. 5. Sherwood Number versus Reynolds number based on pore diameter $D_{p}$. Lines: literature correlations; Symbols: present work.

different authors. This reflects the great variability of both the experimental conditions and the foams physical parameters such as dimensions, porosity, specific surface, wash coat thickness and composition.

Nevertheless our results show that the pressure drop of $\mathrm{KC}$ structures lies on the upper end of published values for foams, while, due to the regularity and the absence of closed pores, one may have expected the opposite. One reason may be that for a given porosity the KC structure presents a higher specific surface area (SSA), as seen in Table 1 . In real foams the surface tension tends to minimize the surface energy by reducing the total surface and accumulating material at the cell nodes.

Also the mass transfer is overestimated. The best comparison of both pressure drop and mass transfer is obtained with works of Giani et al. (2005a) and Groppi et al. (2007) which have studied high porosity foams $(\varepsilon>0.9)$. The reason may be that the geometrical structure of such foams is more similar to a $\mathrm{KC}$ structure. Incera Garrido et al. (2008) reported that foams with low PPI have a significant higher Sherwood number than those of higher PPI. We assume that foams with bigger pore diameters (lower PPI) or porosity may resemble more the geometrical structure of a KC, and thus a better prediction can be achieved by the $\mathrm{KC}$ model. Instead, decreasing the pore size or the porosity will result in less uniform struts with more material accumulation at the struts nodes, resulting in higher discrepancies with the $\mathrm{KC}$ model. This discrepancies mainly affect the SSA. However, the higher SSA may not be the only cause for the higher Sh observed which may be attributed also to the absence of any washcoat model and to the infinite fast model for the surface chemical reactions. The overall reaction speed in real foams is limited by the diffusion within the micro pores of the washcoat. Moreover the non-uniform distribution of the precious metal on the catalyst surface may further limit the reactions even in the transport limited regime.

\subsection{Surface and porosity model}

The foam structure is described by the mean internal pore diameter, $d_{p}$, the mean strut diameter, $d_{s}$, the foam porosity, $\varepsilon$. Several studies, Richardson et al. (2000), Twigg and Richardson (2007), Incera Garrido et al. (2008), Giani et al. (2005a), provide an overview of properties of the used foams. An estimation of $d_{p}$ and $d_{s}$ can be obtained by visual inspection and the porosity can be easily obtain from the density ration $\varepsilon=1-\rho_{\text {foam }} / \rho_{\text {strut }}$, where $\rho_{\text {foam }}$ is obtained by measuring the global volume and weight of the foam sample, and $\rho_{\text {strut }}$ is the density of the foam matrix. The foam performance is also closely related to the specific surface area, SSA. However its measurement is much more cumbersome and uncertain. Thus several relations have been developed modelling the foam structure with cubic cells (Giani et al., 2005a) or with Kelvin cells (tetrakaidecahedra) (Buciuman and KraushaarCzarnetzki, 2003; Inayat et al., 2011a). In particular Inayat et al. revisited the state of the art correlations for different strut morphologies and validated them with experimental data (Inayat et al., 2011a).

\subsubsection{Kelvin cell model}

In the present study it was decided to use the regular stacked Kelvin cells model in the simulations as a representation of the foam structure. This approximation has a series of implications. A Kelvin cell can be characterized by a characteristic length $L_{c}$, the strut diameter $d_{s}$ and the Kelvin cell porosity $\varepsilon$. As a characteristic dimension we chose the side of the cube containing the cell, which can be seen as the "external pore size" defined as the "internal pore size" plus the strut diameter $\left(L_{c}=d_{p}+d_{s}\right)$ (Incera Garrido et al., 2008). Since we deal now with regularly stacked Kelvin cells these three parameters are related by geometrical relationships. Thus only the two can be chosen independently:

$\varepsilon=1-\frac{6 \pi d_{s}^{2} L_{s}}{L_{c}^{3}}=1-\frac{3 \pi}{\sqrt{2}}\left(\frac{d_{s}}{L_{c}}\right)^{2}$

where $L_{S}$ is the strut length given by $L_{S}=\frac{\sqrt{2}}{4} L_{c}$.

For analyzing heat and mass transfer as well as chemical reactive phenomena the quantification of the available surface area is of crucial significance. The proper surface area is the external surface area of the struts as if they were perfectly smooth.

Different approaches have been used to determine the surface area, by estimating the surface area of the polyhedra in question (Twigg and Richardson, 2007; Buciuman and Kraushaar-Czarnetzki, 2003), by assuming similarity with packed beds (Richardson et al., 2000) as well as by using MRI (Incera Garrido et al., 2008). By using the regularly stacked Kelvin cell model for approximating the foam structure in this study an expression for the specific surface area (SSA) has been derived by geometrical reasoning and by assuming that each strut is shared among 3 adjacent Kelvin cells:

$S S A=\frac{24 \pi d_{s} L_{S}}{L_{c}^{3}}=\sqrt{24 \sqrt{2} \pi} \frac{\sqrt{1-\varepsilon}}{L_{c}}=10.33 \frac{\sqrt{1-\varepsilon}}{L_{c}}$

To estimate more precisely the properties of a Kelvin cell structure we build a parametric Computer Aided Design (CAD) model using the OpenSource software Salome. Due to the geometrical scaling, a Kelvin cell structure can be fully characterized by a characteristic cell dimension $\left(L_{c}\right)$ and its ratio with the strut diameter $\left(L_{c} / d_{s}\right)$. By using the build-in functions of Salome we numerically computed the porosity, $\varepsilon$, and the specific surface area, $S S A$, for all regular stacked Kelvin cells in the range $3<L_{c} / d_{s}<29$, where 3 is close to the limit Kelvin cell with the strut diameter equal to the strut length. In Fig. $6, \varepsilon$ and the SSA nondimensionalized by $1 / L_{c}$ are plotted respectively with a black solid and a blue solid line. The choice of plotting $S S A * L_{C}$ instead of $S S A$ is motivated by a dimensional analysis. The porosity is a dimensionless parameter and in an ideal Kelvin cell structure it depends only on the dimensionless ratio $L_{c} / d_{s}$ (Eq. (5)), but SSA has dimension $\left(\mathrm{m}^{-1}\right)$ so it will depend both on the ratio $L_{c} / d_{s}$ and on the characteristic length $L_{c}(\mathrm{~m})$ (Eq. (6)). This consideration and Eq. (6) show that the SSA for all configurations $\left[L_{c} / d_{s}, L_{c}\right]$ can be conveniently represented by the dimensionless specific surface area $S S A * L_{c}$, which depend only on $L_{c} / d_{s}$. The advantage of this choice is that Fig. 6 fully represents all the possible Kelvin cell structures, and it allows us to determine all the four parameters 


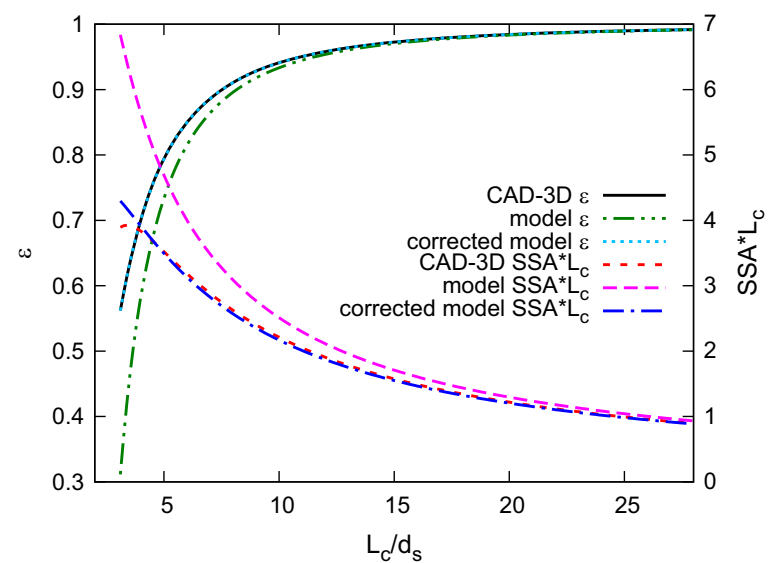

Fig. 6. Porosity and non-dimensional wet surface of a Kelvin Cell as a function of the KC dimension to strut diameter ratio. Relations have been derived numerically from a CAD 3D model, by approximate geometrical models and by correcting the geometrical models to the match the results of the 3D CAD model. (For interpretation of the references to colour in this figure caption, the reader is referred to the web version of this paper.)

$d_{p}=L_{c}-d_{s}, d_{s}, \varepsilon$ and SSA simply by knowing any consistent combination of two of them.

\subsubsection{Kelvin cell corrected model}

The CAD results of the previous section can be used to refine the models proposed in Eqs. (5) and (6). Such models were derived by assuming that in a regular stacked structure each cell con-tributes to the total volume and surface only with 12 struts since each cell has 36 struts and each strut is shared by 3 cells. This was an overestimation since no correction was done for the strut intersection at the structure nodes. In Fig. 6 we compare directly the models with the CAD results and we see that they are in excellent agreement for high $L_{c} / d_{s}$ ratios where the struts are thin. However the deviation of the model became significant when the strut length is only 4 times its diameter or less and a correction is necessary for this range. By noting that the volume and the surface of the intersections do not depend on the length strut but only on the strut diameter we conclude that the corrections on $\varepsilon$ and $S S A * L_{c}$ need to have the form of $K_{1}\left(d_{S} / L_{c}\right)^{3}$ and $K_{2}(1-\varepsilon)$, where $K_{1}$ and $K_{2}$ are chosen in order to match the CAD numerical results. The corrected form of the models becomes

$\varepsilon=1-\frac{3 \pi}{\sqrt{2}}\left(\frac{d_{s}}{L_{c}}\right)^{2}+7.54\left(\frac{d_{s}}{L_{c}}\right)^{3}$

$S S A=10.33 \frac{\sqrt{1-\varepsilon}}{L_{C}}-5.8 \frac{1-\varepsilon}{L_{C}}$

Fig. 6 shows the improvements of the corrected models.

\subsection{Pressure drop}

As expected the pressure drop increases exponentially with the velocity. Fig. 7 shows the pressure drop $\Delta P / L(\mathrm{~Pa} / \mathrm{mm})$ plotted against the internal velocity $u_{i}$, the average velocity inside the foam, computed as the surface velocity divided the porosity:

$u_{i}=u / \varepsilon$. The two arrows in the figure indicate the $\Delta P / L$ behaviour with the PPI and the porosity, $\varepsilon$. Increasing the PPI of the KC structure results in a strong increase of the pressure drop. In our simulation pressure increases up to 5 times when passing from 10 PPI to 44 PPI. Such increase is obtained at constant porosity and inflow velocity and is mainly due to an increased SSA. At the same time, as expected, $\Delta P / L$ decreases with increasing porosity. In Fig. 7 we see that the data with high porosity $(\varepsilon=0.89)$ have a reduction of about $50 \%$ (minimum $45.8 \%$, maximum $52.4 \%$ )

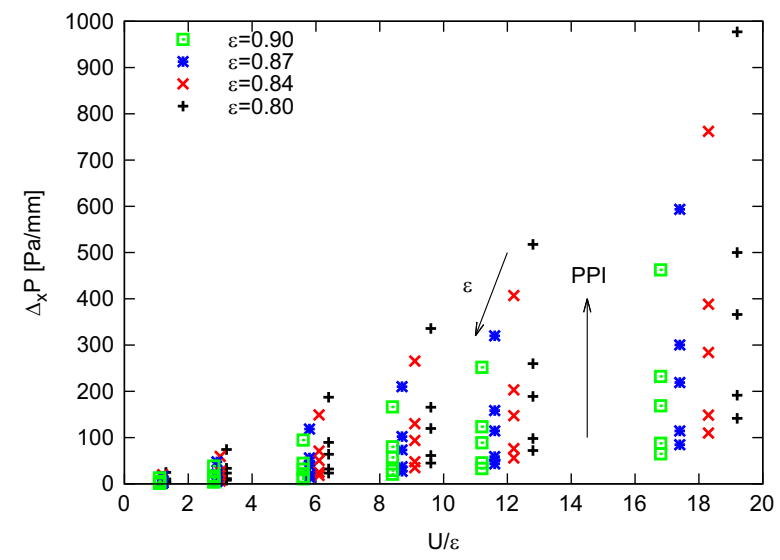

Fig. 7. Pressure drop per unit length in $\mathrm{Pa} / \mathrm{mm}$ versus internal velocity $u_{i}=u / \varepsilon$.

compared to the case with $\varepsilon=0.78$ and same PPI and inflow velocity (thus similar internal velocities).

\subsubsection{Pressure drop model}

By elaboration the work of Du Plessis et al. (1994) and Ahmed et al. (2011) we model the pressure drop on a Kelvin cell structure. We start from the consideration that in a single cell the pressure gradient is given by the total drag force on the cell divided by the fluid volume:

$-\frac{d p}{d x}=\frac{F_{D}}{\varepsilon L_{c}^{3}}$

In foam catalysts the Reynolds number is usually much higher than 1, where Darcy's law, which is derived for creeping flows $(\operatorname{Re} \ll 1)$, does not apply. In such a regime the inertial contribution will be important. Thus our choice is to rescale pressure and drag forces by an inertial term $\left(\rho u^{2} / 2\right)$ and by following the classical approach for the drag force modelling, we define a drag coefficient $C_{D}$ such that

$F_{D}=C_{D} \frac{\rho u_{p}^{2}}{2} A_{w}$

where $\rho$ is the fluid density, $u_{p}$ is the average fluid velocity inside the pore (Ahmed et al., 2011) and $A_{w}$ is the wet surface. An expression for the average pore velocity can be derived from simple consideration on the flow rate conservation. Assuming incompressible flows, the flow rate inside the Kelvin cell structure is given by $u_{p} A_{p}$, where $A_{p}$ is the cell cross section area. Since the volumetric flow rate at the inflow is equal to $u L_{c}^{2}$ we can express $u_{p}$ as $u_{p}=u L_{c}^{2} / A_{p}$. We now introduce the concept of tortuosity $(\chi)$ as the ratio between the effective path of the fluid lines and the longitudinal length. For a porous media the tortuosity has been defined by Du Plessis et al. (1994) and can be expressed as (Du Plessis et al., 1994) (see also Appendix A, Eq. (A.3))

$\chi=\frac{\varepsilon L_{c}^{2}}{A_{p}}$.

By using the tortuosity (Eq. (11)) $u_{p}$ is given by

$u_{p}=u_{\varepsilon}^{\chi}$

Finally by combining Eqs. (9), (10), (12) and by remembering that the $S S A=A_{w} / L_{c}^{3}$ we obtain

$-\frac{d p}{d x}=S S A \frac{\rho u^{2}}{2} \frac{\chi^{2}}{\varepsilon^{3}} C_{D}$.

It is interesting to highlight where the terms of Eq. (13) come from in order to understand their physical origin in this model. 

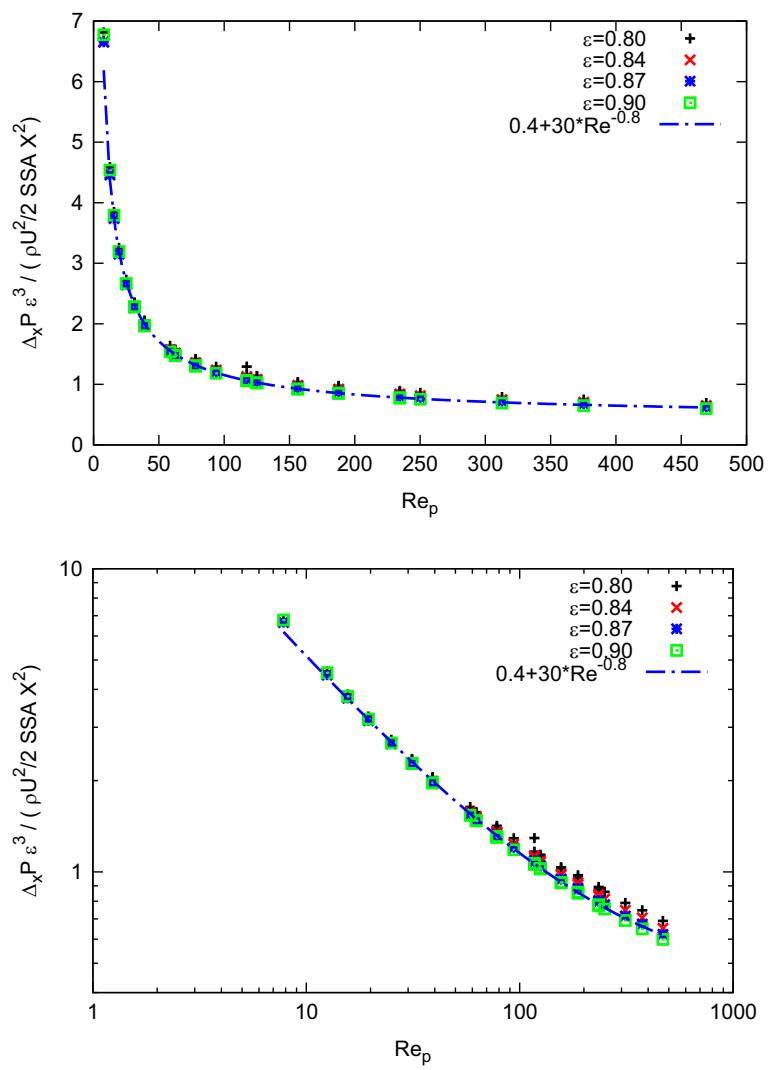

Fig. 8. Non dimensional coefficient $C_{D}$ defined by Eq. (13) versus pore Reynolds number. Top: linear plot; bottom: log-log plot.

The tortuosity and part of the porosity dependence $\left(\chi^{2} / \varepsilon^{2}\right)$ come from the fact that the relevant velocity to determine the drag and the pressure drop is the average velocity inside the pore $u_{p}$ (Eq. (12)), while for convenience we express the pressure in terms of the external velocity $u$. The SSA come from the cell wet area divided by the cell volume and indicate that the effect of the drag force is distributed over the full volume of the KC structure down to the single cell unit (Eq. (9)). Finally the third dependence on the porosity $(1 / \varepsilon)$ comes from the fact that the drag force distribution happens only on the fraction of the fluid volume (Eq. (9)).

\subsubsection{Model validation}

In Fig. 8 the coefficient $C_{D}$ is plotted versus the Reynolds number for the full set of our data. All the data follow a single line confirming the validity of the scaling in Eq. (13). Only a small deviation from the line is observed between different porosity sets. Thus in our case $C_{D}$ depends mainly on the Reynolds number with only small residual dependence on the porosity. This result may not be general and need to be confirmed on real foams. The reason is that different from the $\mathrm{KC}$, foams cannot be geometrically rescaled adding an extra parameter in the geometrical characterization. In other words it needs to be confirmed if maintaining explicitly the dependence on SSA, $\chi$ and $\varepsilon$ in the scaling of the pressure, as in Eq. (13), instead of expressing everything in terms of porosity and $D_{p}$ will be enough to accurately describe all possible parameter combinations of real foams. Moreover, since the model is based on the drag one may expect that the leading Reynolds number would have been based on the strut diameter $d_{S}$, similarly for the case of the flow over a cylinder. However the best data convergence is obtained by using the Reynolds number based on the cell dimension $L_{c}=d_{p}+d_{s}$. This observation is consistent with the experimental results of Incera Garrido et al. (2008). (Note that Incera Garrido et al. use the pore diameter defined as the inner pore diameter plus the strut diameter, like our $L_{c}$.) A similar conclusion was obtained by Yazdchi and Luding (2012) who study the flow through fibrous material observed that the results scale with the gap Reynolds number, based on mean value of the averaged 2nd nearest neighbour surface to surface fibre distance, rather than with the Reynolds number based on the fibre diameter.

In Fig. 8 a good fit for $C_{D}$ is obtained by the expression:

$C_{D}=0.4+\stackrel{\frac{30}{\operatorname{Re}^{0.8}}}{\text {. }}$

If we convert Eq. (13) to the Darcy-Forchheimer

equatio $p=\frac{\rho}{k} u+\frac{\rho}{k_{1}} u^{2}$

$C_{D}$ should have a form of $a+b /$ Re to have permeability coefficients $k$ and $k_{1}$ independent of the flow rate. The 0.8 exponent in Eq. (14) implies a residual velocity dependence on the permeability constant $k$. Fourie and Du Plessis (2002) had already argued this dependence on the inertial permeability constant $k_{1}$. The two observations are consistent since in their analytical analysis the shear factor was split into two terms, one for low Reynolds numbers and one for high Reynolds numbers. They modelled the high Reynolds number term using the drag coefficient of a cylinder in cross flow and assume it to be equivalent to the inertial term of the Darcy-Forchheimer equation. Doing so they basically added a viscous correction to the inertial term.

The common conclusion from this two approaches is that $k$ and/or $k_{1}$ have a residual velocity dependence which may contribute to the difficulties in fitting the experimental data.

\subsection{Mass transfer}

In this section we want to characterize the mass transfer properties of the Kelvin cell structures. In Fig. 9 we plot the total $\mathrm{CH}_{4}$ conversion versus the pressure drop per unit of length $\Delta P / L_{R}$. Independent of porosity or PPI all the points of the same velocity lay on the same line. Thus, at fixed inflow velocity the conversion increases only with pressure and it is independent of porosity and PPI. Lines characterized by higher velocity are shifted to lower conversions, so for fixed pressure gradients increasing the velocity will decrease the conversion. By decreasing the velocity, the velocity-line is pushed toward the low-pressure/high-conversion regime. All the cases with low velocities $(1 \mathrm{~m} / \mathrm{s})$ have reached full conversion so the conversion became independent of the pressure. It has to be remembered that in this plot we compare the global conversion rate of reactors of different sizes, since the number of cells in the structure $(2 \times 2 \times 4)$ is kept constant. The highest

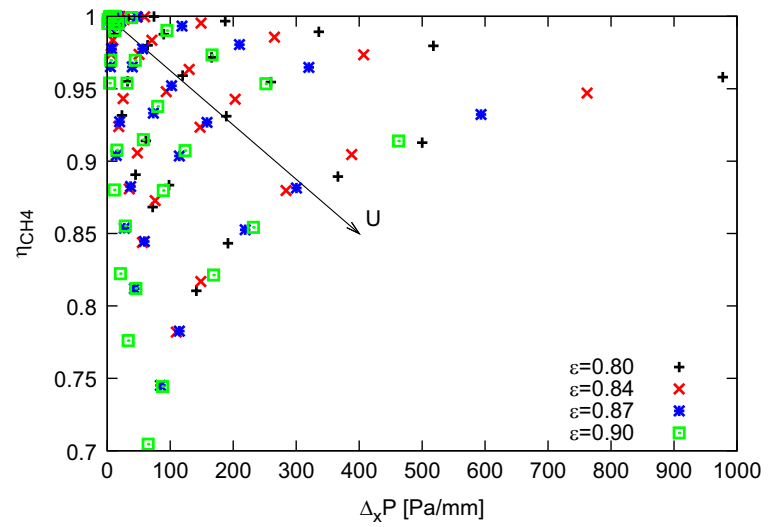

Fig. 9. Conversion rate of methane $\eta_{\mathrm{CH} 4}$. 


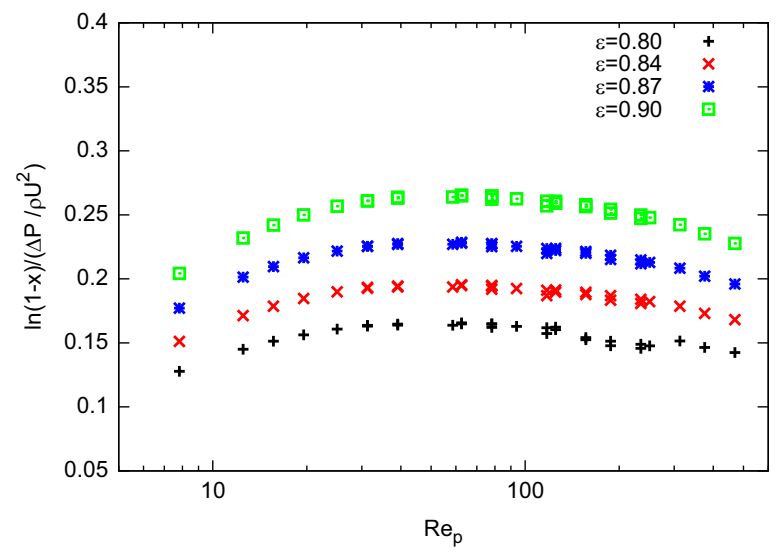

Fig. 10. Performance coefficient $I$ (Eq. (16)) versus pore Reynolds number.

pressure gradients are obtained for foams with the highest PPI because a similar pressure drop is generated in a much smaller space.

To evaluate the performance of foam structures Giani et al. (2005a) defined a dimensionless trade-off index as

$I=\frac{-\ln (1-\eta)}{\Delta P /\left(\rho u^{2}\right)}$

where $\eta$ is the global conversion. Best structures have higher index since they give higher conversion rate for a given pressure drop. The index I for all porosities is plotted in Fig. 10 versus the Reynolds number. From the figure we see that higher performance is obtained by higher porosities. Moreover the index peaks for all cases around Reynolds number 70 but its value stays somehow stable in the range of Reynolds number [40:100].

\subsubsection{Lévêque analogy}

Recently Martin (2002) presented a new analogy between pressure drop and mass transfer that can be used in heat exchangers or in other periodic or randomly periodic arrangements like pack beds. It is based on the generalized Lévêque equation

$\frac{N u}{\operatorname{Pr}^{1 / 3}}=\frac{S h}{S c^{1 / 3}}=0.404\left(2 x_{f} \cdot H g \cdot \frac{D_{h}}{L}\right)^{1 / 3}$,

where $\mathrm{Hg}$ is a dimensionless number, the Hagen number, related to the driving force of the flow defined as

$H g=\frac{\Delta P}{\Delta x} \cdot \frac{D_{h}^{3}}{\rho \nu^{2}}$,

where $x_{f}$ is the frictional factor and $D_{h}$ is the hydraulic diameter (or $D_{p}$ for our case). The analogy was confirmed for catalytic foams by Incera Garrido et al. (2008) which proposed the correlation:

$S h_{I G}=0.62 \cdot\left(\frac{D_{p}}{0.001 m}\right)^{0.48} \cdot \varepsilon^{2.34} \cdot H g^{0.31} \cdot S c^{1 / 3}$

In Fig. 11 we plot the Sherwood number versus the Hagen number $\mathrm{Hg}$ and we compared it with Eq. (19). Our results do not show any dependence on the $D_{p}$. And, as in Section 3.1, the best agreement is obtained by applying Incera Garrido's correlations with low PPI correlations, suggesting that low PPI real foams resemble more closely an idealized KC structure. Another possible point to be investigated is the foam anisotropy. In a later paper Incera Garrido and Kraushaar-Czarnetzki (2010) modify Eq. (19) by substituting the $D_{p}$ dependence with an anisotropy factor based on the average pore dimension on each direction. The fact that all our KC structures are isotropic may explain why we do not see such a dependence. Further investigations may clarify this point.

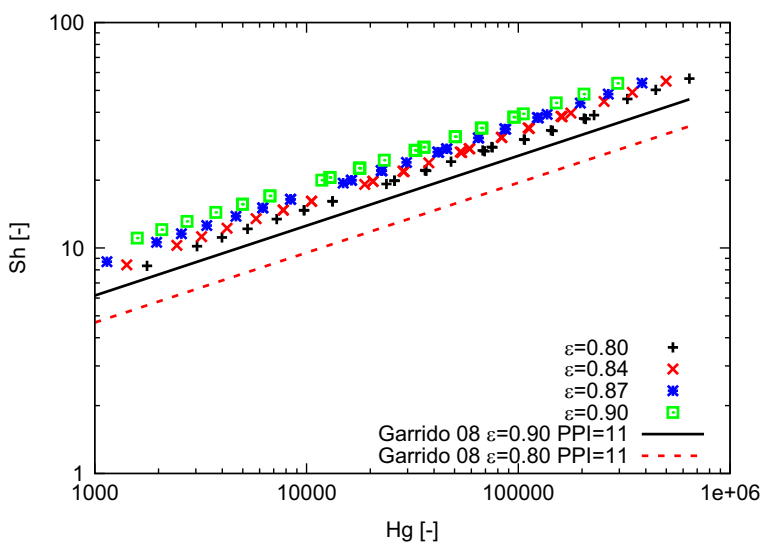

Fig. 11. Sherwood number versus dimensionless pressure drop, $\mathrm{Hg}$ (Eq. (18)).

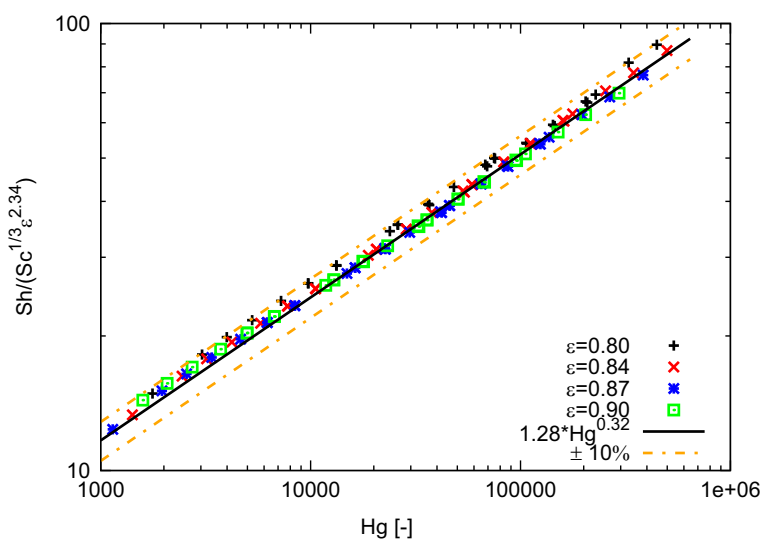

Fig. 12. Correlated Sherwood number (Eq. (20)) versus dimensionless pressure drop, $\mathrm{Hg}$.

However the remaining dependence on the Hagen number, $\mathrm{Hg}$, and on porosity, $\varepsilon$, of our results in Fig. 11 is in very good agreement with the correlation in Eq. (19). In Fig. 12 the Sherwood number is scaled by porosity, $S h /\left(S c^{1 / 3} \varepsilon^{2.34}\right)$, according to Eq. (19). All points fit the correlation

$\frac{S h}{S c^{1 / 3} \varepsilon^{2.34}}=1.28 \mathrm{Hg}^{0.32}$

within an accuracy of $10 \%$. On one hand the $\mathrm{Hg}^{0.32}$ term is very close to the $\mathrm{Hg}^{0.31}$ predicted by Eq. (19), on the other hand, for the same reasons discussed in Section 3.1, the fitting parameter, 1.28, is higher.

\section{Conclusions}

A parametric study of the momentum and mass transfer in randomized Kelvin cell structures have been performed. Infinite fast oxidation of methane was assumed to be on the fluid-solid boundary. Washcoat diffusion was neglected. The main conclusions and achievements of the work are

- Accurate correlations for SSA and porosity of Kelvin cells are derived using geometrical considerations and matching them with a CAD model.

- By elaborating the work of Du Plessis et al. (1994) and Ahmed et al. (2011) we develop a model that highlights the main 
physical parameters and their origin. The model requires to find 3 parameters by fitting the foam drag coefficient with experimental or numerical data.

- Our results on the Kelvin cell structure show a higher mass transfer compared to the $S h$ correlations presented in the literature. In particular our results are about 20\% higher than that predicted by the correlation of Groppi et al. (2007). We attribute this to the different geometrical properties of the foams and to the assumptions made.

- Due to the geometrical similitude of the Kelvin cell structures our pressure and Sherwood number correlations do not present the pore diameter $D_{p}$ dependence reported by Incera Garrido et al. (2008).

- The Sherwood number is correlated with the Hagen number (dimensionless pressure drop) within a 10\% error. The correlation was qualitatively in very good agreement with that observed by Incera Garrido and Kraushaar-Czarnetzki (2010).

\section{Nomenclature}

$\chi \quad$ tortuosity (dimensionless)

$\eta \quad$ conversion rate (dimensionless)

$\nu \quad$ kinematic viscosity $\left(\mathrm{m}^{2} / \mathrm{s}\right)$

$D_{p} \quad$ external pore diameter $D_{P}=d_{p}+d_{s}=L_{c}(\mathrm{~m})$

$\mathrm{Hg} \quad$ Hagen number $\mathrm{Hg}=\Delta P / \Delta x \cdot D_{h}^{3} / \rho \nu^{2}$ (dimensionless)

$L_{R} \quad$ reactor length $(\mathrm{m})$

$\operatorname{Re}_{p} \quad$ pore Reynolds number $\operatorname{Re}_{p}=\frac{D_{p} u}{\nu}$ (dimensionless)

$u \quad$ inflow velocity $(\mathrm{m} / \mathrm{s})$

$Y_{X} \quad$ mass fraction of species $X$ (dimensionless)

$d_{p} \quad$ internal pore diameter $(\mathrm{m})$

$d_{s} \quad$ strut diameter $(\mathrm{m})$

$\varepsilon \quad$ porosity (dimensionless)

$L_{c} \quad$ KC characteristic length $L_{c}=d_{p}+d_{s}=D_{p}(\mathrm{~m})$

$L_{S} \quad$ strut length $L_{S}=\frac{\sqrt{2}}{4} L_{c}(\mathrm{~m})$

SSA specific surface area $\left(\mathrm{m}^{2} / \mathrm{m}^{3}\right)$

KC Kelvin cell

PPI pore per inch

\section{Acknowledgements}

The authors gratefully acknowledge financial support from Swiss Kompetenzzentrum für Energie und Mobilität CCEM via Project Number 704 and from the Italian Ministry of Education, University and Research, Rome (MIUR, Progetti di Ricerca Scientifica di Rilevante Interesse Nazionale, prot. 2010XFT2BB), within the project IFOAMS: Intensification of Catalytic Processes for Clean Energy, Low-Emission Transport and Sustainable Chemistry using Open-Cell Foams as Novel Advanced Structured Materials.

\section{Appendix A. Tortuosity derivation for Kelvin cells}

In a porous media the tortuosity is defined as the ratio between the effective length $\left(L_{e}\right)$ of the pathlines to the stream-wise length scale of the porous structure $\left(L_{c}\right)$ (Du Plessis et al., 1994):

$\chi \equiv \frac{L_{e}}{L_{c}} \quad$ with $L_{e}=\frac{\varepsilon L_{c}^{3}}{A_{p}}$

Here the effective length is computed as the fluid volume in a Kelvin cell $\left(\varepsilon L_{c}^{3}\right)$ divided by the fluid cross section area in the cell inlet. In first approximation this area is equivalent to

$A p=L_{c}^{2}-4 d_{s} L_{s}$
Substitutingq. (A.2) in Eq. (A.1), rearranging and substituting $L_{S} / L_{c}$ $=2 / 4$ we obtain

$\chi=\frac{\varepsilon L_{c}^{2}}{A_{p}}=\frac{\varepsilon}{1-\sqrt{2} d_{s} / L_{c}}$

From Eqs. (5) and (6) we can express $d_{s} / L_{c}$ as

$\left.\frac{d_{s}}{L_{c}}=\frac{\sqrt{2}}{3 \pi}\right)^{1 / 2} \sqrt{1-\varepsilon}=\frac{S S A L_{c}}{6 \sqrt{2} \pi^{3 / 4}}$.

Finally by combining Eqs. (A.3) and (A.4) the tortuosity can expressed as

$\chi=\frac{\varepsilon}{1-\frac{2^{3 / 4}}{(3 \pi)^{1 / 2}} \sqrt{1-\varepsilon}}=\frac{\varepsilon}{1-\frac{S S A L_{c}}{6 \pi^{3 / 4}}}$

\section{References}

Ahmed, J., Pham-Huu, C., Edouard, D., 2011. A predictive model based on tortuosity for pressure drop estimation in 'slim' and 'fat' foams. Chem. Eng. Sci. 66 (20), 4771-4779.

Bai, M., Chung, J., 2011. Analytical and numerical prediction of heat transfer and pressure drop in open-cell metal foams. Int. J. Therm. Sci. 50 (6), 869-880.

Boomsma, K., Poulikakos, D., Ventikos, Y., 2003. Simulations of flow through open cell metal foams using an idealized periodic cell structure. Int. J. Heat Fluid Flow 24 (6), 825-834.

Buciuman, F., Kraushaar-Czarnetzki, B., 2003. Ceramic foam monoliths as catalyst carriers. 1. Adjustment and description of the morphology. Ind. Eng. Chem. Res. 42 (9), 1863-1869.

Dimopoulos Eggenschwiler, P., Tsinoglou, D., Seyfert, J., Bach, C., Vogt, U., Gorbar,

M.,

2009. Ceramic foam substrates for automotive catalyst applications: fluid mechanic analysis. Exp. Fluids 47 (2), 209-222.

Du Plessis, P., Montillet, A., Comiti, J., Legrand, J., 1994. Pressure drop prediction for flow through high porosity metallic foams. Chem. Eng. Sci. 49 (21), 3545-3553. Edouard, D., Lacroix, M., Pham, C., Mbodji, M., Pham-Huu, C., 2008. Experimental measurements and multiphase flow models in solid SiC foam beds. AIChE J. 54

(11), 2823-2832.

Evans, A.G., Hutchinson, J.W., Ashby, M.F., 1998. Cellular metals. Curr. Opin. Solid State Mater. Sci. 3 (3), 288-303.

Fourie, J., Du Plessis, J., 2002. Pressure drop modelling in cellular metallic foams Chem. Eng. Sci. 57 (14), 2781-2789.

Gaiser, G., Oesterle, J., Braun, J., Zacke, P., 2003. The Progressive Spin InletHomogeneous Flow Distributions under Stringent Conditions. SAE Technical Papers.

Giani, L., Groppi, G., Tronconi, E., 2005a. Mass-transfer characterization of metallic foams as supports for structured catalysts. Ind. Eng. Chem. Res. 44 (14) 4993-5002.

Giani, L., Groppi, G., Tronconi, E., 2005b. Heat transfer characterization of metallic foams. Ind. Eng. Chem. Res. 44 (24), 9078-9085.

Groppi, G., Giani, L., Tronconi, E., 2007. Generalized correlation for gas/solid masstransfer coefficients in metallic and ceramic foams. Ind. Eng. Chem. Res. 46 (12), 3955-3958.

Habisreuther, P., Djordjevic, N., Zarzalis, N., 2009. Statistical distribution of residence time and tortuosity of flow through open-cell foams. Chem. Eng. Sci. 64 (23), 4943-4954.

Huu, T., Lacroix, M., Pham Huu, C., Schweich, D., Edouard, D., 2009. Towards a more realistic modeling of solid foam: use of the pentagonal dodecahedron geome-try. Chem. Eng. Sci. 64 (24), 5131-5142.

Inayat, A., Freund, H., Zeiser, T., Schwieger, W., 2011a. Determining the specific surface area of ceramic foams: the tetrakaidecahedra model revisited. Chem. Eng. Sci. 66 (6), 1179-1188.

Inayat, A., Schwerdtfeger, J., Freund, H., Körner, C., Singer, R., Schwieger, W., 2011b. Periodic open-cell foams: pressure drop measurements and modeling of an ideal tetrakaidecahedra packing. Chem. Eng. Sci. 66 (12), 2758-2763.

Incera Garrido, G., Kraushaar-Czarnetzki, B., 2010. A general correlation for mass transfer in isotropic and anisotropic solid foams. Chem. Eng. Sci. 65 (6) 2255-2257.

Incera Garrido, G., Patcas, F., Lang, S., Kraushaar-Czarnetzki, B., 2008. Mass transfer and pressure drop in ceramic foams: a description for different pore sizes and porosities. Chem. Eng. Sci. 63 (21), 5202-5217.

Koltsakis, G.C., Katsaounis, D.K., Samaras, Z.C., Naumann, D., Saberi, S., Böhm, A., Markomanolakis, I., Development of Metal Foam Based Aftertreatment System on a Diesel Passenger Car. SAE Technical Papers, 2008.

Krishnan, S., Murthy, J., Garimella, S., 2006. Direct simulation of transport in opencell metal foam. J. Heat Transf. 128 (8), 793-799.

Lacroix, M., Nguyen, P., Schweich, D., Pham Huu, C., Savin-Poncet, S., Edouard,

D.,

2007. Pressure drop measurements and modeling on SiC foams. Chem. Eng. Sci. 62 (12), 3259-3267. 
Martin, H., 2002. The generalized Lévêque equation and its practical use for the prediction of heat and mass transfer rates from pressure drop. Chem. Eng. Sci. 57 (16), 3217-3223.

Martin, A.P., Will, N.S., Bordet, A., Cornet, P., Gondoin, C., Mouton, X., Effect of Flow Distribution on Emissions Performance of Catalytic Converters. SAE Technical Papers, 2008.

Richardson, J., Peng, Y., Remue, D., 2000. Properties of ceramic foam catalyst supports: pressure drop. Appl. Catal. A: Gen. 204 (1), 19-32.

Richardson, J., Remue, D., Hung, J.-K., 2003. Properties of ceramic foam catalyst supports: mass and heat transfer. Appl. Catal. A: Gen. 250 (2), 319-329.
Twigg, M., Richardson, J., 2007. Fundamentals and applications of structured ceramic foam catalysts. Ind. Eng. Chem. Res. 46 (12), 4166-4177.

Yazdchi, K., Luding, S., 2012. Towards unified drag laws for inertial flow through fibrous materials. Chem. Eng. J. 207-208, 35-48.

Zygourakis, K., 1989. Transient operation of monolith catalytic converters: a

twodimensional reactor model and the effects of radially nonuniform flow distributions. Chem. Eng. Sci. 44 (9), 2075-2086. 